\title{
Observations of large-scale fluid transport by laser-guided plankton aggregations ${ }^{\text {a) }}$
}

\author{
Monica M. Wilhelmus ${ }^{1}$ and John O. Dabiri' ${ }^{2}$ \\ ${ }^{1}$ Mechanical Engineering, California Institute of Technology, Pasadena, \\ California 91125, USA \\ ${ }^{2}$ Graduate Aeronautical Laboratories and Bioengineering, California Institute of \\ Technology, Pasadena, California 91125, USA
}

(Received 13 June 2014; accepted 27 August 2014; published online 30 September 2014)

\begin{abstract}
Diel vertical migration of plankton has been proposed to affect global ocean circulation to a degree comparable to winds and tides. This biomixing process has never been directly observed, however, due to the inability to predict its occurrence in situ or to reproduce it in a laboratory setting. Furthermore, it has been argued that the energy imparted to the ocean by plankton migrations occurs at the scale of individual organisms, which is too small to impact ocean mixing. We describe the development of a multi-laser guidance system that leverages the phototactic abilities of plankton to achieve controllable vertical migrations concurrently with laser velocimetry of the surrounding flow. Measurements in unstratified fluid show that the hydrodynamic interactions between neighboring swimmers establish an alternate energy transfer route from the small scales of individually migrating plankton to significantly larger scales. Observations of laser-induced vertical migrations of Artemia salina reveal the appearance of a downward jet, which triggers a Kelvin-Helmholtz instability that results in the generation of eddy-like structures with characteristic length scales much larger than the organisms. The measured energy spectrum is consistent with these findings and indicates energy input at large scales, despite the small individual size of the organisms. These results motivate the study of biomixing in the presence of stratification to assess the contribution of migrating zooplankton to local and global ocean dynamics. The laser control methodology developed here enables systematic study of the relevant processes. (c)2014 AIP Publishing LLC. [http://dx.doi.org/10.1063/1.4895655]
\end{abstract}

\section{INTRODUCTION}

The bulk of ocean biomass is composed of zooplankton species such as krill, which aggregate in dense groups within the water column. ${ }^{1}$ Mostly idle at depth, these densely packed swarms migrate vertically towards the surface to feed following the cues of sunrise and sunset. ${ }^{2,3}$ It has been hypothesized that these diel vertical migrations (DVM) can have a substantial impact on fluid transport in the ocean. ${ }^{3}$ However, the resulting local and global effects of these collective motions have not been quantified. ${ }^{4}$ Specifically, it remains an open question whether DVM induce fluid transport mechanisms relevant to the physical and biogeochemical structure of the ocean.

By swimming over large vertical distances, small organisms have the potential to impact naturally occurring nutrient and density gradients. Locally, nutrients are unevenly distributed throughout the water column primarily due to the negative buoyancy of most particulate organic matter and remineralization by bacteria at depth. ${ }^{5}$ In the near surface region, the concentration of nutrients is limited by biological consumption, whereas at increasing depths light absorption limits biological activity

\footnotetext{
a) This paper was presented as an invited talk at the 66th Annual Meeting of the APS Division of Fluid Dynamics, 24-26 November 2013, Pittsburgh, Pennsylvania, USA.
} 
leading to an increase in nutrient concentration. ${ }^{6}$ Moreover, in the oxygen-depleted regions of the deep ocean, bacteria transform inorganic matter into absorbable macromolecules such as nitrate and phosphorous, further increasing the concentration of nutrients at depth. Nutrient replenishment near the ocean surface occurs via terrestrial runoff and via transport from nutrient-rich regions at depth resulting from vertical mixing. By inducing vertical fluid motion, swimming organisms may actively modify the distribution of nutrients within their natural environment, and thereby enhance biological production that occurs throughout the ocean.

Local variations in salinity and temperature with depth superimpose a stable density gradient onto the nutrient distribution of the water column, which impedes fluid and nutrient transport across isopycnals, i.e., surfaces of constant density. On a global scale, the stability of the density gradient along with global ocean circulation dictate nutrient and energy transport in the ocean. The global overturning circulation, known as the Meridional Overturning Circulation (MOC), connects the ocean basins and transfers heat throughout the ocean. On average, the MOC transports warm water from the equator to the poles, where convective processes lead to a decrease in the potential energy of the ocean by converting warm water masses into colder, denser fluid that sinks into the deep ocean. In order to complete the MOC, mechanical energy is needed to subsequently raise the potential energy of the system via vertical mixing across the stable stratification. The currently known primary drivers for this vertical transport are winds and tides, which collectively provide approximately 2 trillion watts (TW) of mechanical energy flux. ${ }^{7}$ This influx of energy becomes available throughout the water column as a result of intermittent internal wave generation and breaking.

Organisms in the ocean have a reservoir of chemical energy supplied by phytoplankton near the ocean surface at a rate of approximately $60 \mathrm{TW}^{2}$ If even $1 \%$ of this power contributes to largescale fluid mixing in the ocean via an efficient hydrodynamic conversion to mechanical energy, the contribution of swimming organisms to the MOC energy budget could be comparable to the input of winds and tides. Thus, in addition to its potential role in local ecosystems, biogenic fluid transport could have a significant contribution to global ocean dynamics.

Identifying the underlying fluid transport mechanisms during DVM of zooplankton aggregations is crucial to assessing the potential role of swimming animals in fluid dynamic processes in the ocean. Currently, swimming organisms are known to transport fluid by turbulent wake mixing and induced fluid drift. ${ }^{8,9}$

In the process of turbulent wake mixing, unsteady swimming by organisms sets fluid into motion by generating eddy-like structures at scales comparable to the organism appendages. This energy is expected to cascade to smaller scales until it is dissipated as heat by viscosity. During this energy cascade, fluid properties and nutrients can be transported on length scales commensurate with the size of the formed eddies. Field measurements, aiming to quantify biogenic mixing with respect to background ocean levels, showed elevated rates of turbulent kinetic energy (TKE) dissipation in the vicinity of a DVM of krill in Saanich Inlet, British Columbia. ${ }^{3}$ Within a span of 10-15 min, TKE dissipation rates increased by 3-4 orders of magnitude compared to background ocean turbulence, reaching values of $10^{-5}-10^{-4} \mathrm{~W} \mathrm{~kg}^{-1}$. These measurements agreed with theoretical estimates for the dissipation due to turbulent wake transport. ${ }^{1,2}$ However, a subsequent analysis noted that higher TKE dissipation rates do not necessarily imply enhanced fluid mixing. ${ }^{8}$ Hence, an important metric to determine the impact of biogenic mixing is its efficiency, rather than the rate at which energy is dissipated.

Biogenic mixing efficiency is commonly estimated via the flux Richardson number $R_{f}$, which relates the change in potential energy of the water column via vertical mixing to the total amount of kinetic energy expended by a given mixing process: ${ }^{11}$

$$
R_{f}=\frac{\Delta P E}{\Delta K E}
$$

The general expression in Eq. (1) can be rewritten as a ratio of length scales in order to determine the scales at which biomixing processes must introduce energy into the flow in order to achieve efficient mixing. ${ }^{8}$ This is done by applying Richardson's four-thirds law to relate $R_{f}$ to an eddy 
diffusivity coefficient $K$, where ${ }^{10,11}$

$$
K \approx \varepsilon^{1 / 3} L^{4 / 3},
$$

and

$$
K=\frac{R f}{\left(1-R_{f}\right)} \frac{\varepsilon}{N^{2}}
$$

In Eqs. (2) and (3), $\varepsilon$ is the turbulent kinetic energy dissipation rate, $L$ is the integral length scale, and $N$ is the Brunt-Väisälä buoyancy frequency, which is a measure of the stability of the stratification. By combining Eqs. (2) and (3), the biomixing efficiency $\Gamma$ may be expressed as

$$
\Gamma=\frac{R_{f}}{1-R_{f}} \approx L^{4 / 3}\left(\frac{N^{2}}{\varepsilon^{2 / 3}}\right),
$$

where the last factor can be rewritten in terms of the Ozmidov buoyancy length scale, which characterizes the length scale over which density changes in the ocean ${ }^{8}$

$$
B=\sqrt{\frac{\varepsilon}{N^{3}}} .
$$

This leads to the four-thirds scaling of the mixing efficiency as

$$
\begin{array}{ccl}
\Gamma \approx\left(\frac{L}{B}\right)^{4 / 3} & L<B \\
\Gamma \approx \gamma_{0} & L \geq B,
\end{array}
$$

where $\gamma_{0}$ is the maximum mixing efficiency, which has been estimated to reach a limit of $0.2^{8,10,12}$ (but, see recent work by Ref. 34). A consequence of Eq. (6) is that the size of fluid structures generated by the motion of migrating organisms must approach the Ozmidov length scale in order for biogenic mixing to be efficient.

Field measurements of individual swimming jellyfish suggested a mechanism that would enable organisms to transport fluid on length scales much larger than the appendage size, and thereby achieve efficient mixing. ${ }^{9}$ This mechanism of induced drift has its origins in studies of fluid transport in potential flow. ${ }^{13}$ The volume of fluid carried by an object by the induced drift mechanism is dependent on body shape rather than size and is enhanced by viscosity. ${ }^{9}$ This suggests that organisms swimming at low Reynolds number may transport fluid on scales larger than their characteristic body size and perhaps even greater than the Ozmidov scale, which would lead to efficient mixing. ${ }^{14}$

Recent numerical studies have investigated the concept of biogenic mixing by using models of individual swimming organisms in the Stokes flow limit (e.g., Ref. 15). These models, which capture only the far-field flow, have concluded that the contribution of small swimming animals to ocean mixing is negligible. The apparent contradiction with experimental results suggests that in order to accurately capture the induced fluid drift generated by a real swimmer, both the near- and the far-field flows need to be considered in the analysis.

While previous experiments ${ }^{16}$ as well as the aforementioned numerical studies have focused on the fluid transport induced by individual swimmers, it remains an open question how turbulent wake transport and induced drift are affected by the presence of multiple swimming organisms migrating in close proximity, as occurs during DVM. It is possible that additional mixing mechanisms become relevant during collective motions, as in bioconvection. ${ }^{17,18}$ Furthermore, a simplified model of multiple swimming animals suggests that vertical transport in a stably stratified fluid depends on the arrangement of the swimmers within the group. ${ }^{19}$ Other factors such as the swimming mode of the organisms and the number density of animals within the aggregations are anticipated to affect fluid transport as well.

Recent efforts have been made to obtain field measurements that encompass in situ animal behavior as well as ambient ocean conditions. However, direct shipboard measurements rely on the fortuitous presence of migrating zooplankton, and such observations have proven difficult to repeat. ${ }^{20}$ 
On the other hand, existing numerical studies have yet to overcome the computational expense of modeling the near-field flow of an aggregation of small swimmers migrating vertically. Laboratory experiments have the potential to bridge the gap between field measurements and numerical models by enabling controlled studies of biogenic mixing, but the ability to control vertical migrations of plankton in a laboratory setting has proven elusive. ${ }^{21}$

In this study, fluid transport in an unstratified medium is addressed in an effort to identify the fundamental fluid transport mechanisms that occur during biogenic mixing. Our objective is to identify the transport processes that emerge from collective vertical migrations of small zooplankton and to determine the length scales at which the mechanical energy is input to the fluid during DVM. Controlled vertical migrations of Artemia salina (Linnaeus, 1758) are achieved in a laboratory setting by exploiting the phototactic behavioral response of the organisms to various wavelengths of light. A newly developed laser guidance system is used to control the swimming speed and animal spacing in an aggregation. Evaluation of the generated flow structures is accomplished by implementing two-dimensional particle image velocimetry (PIV). The measured kinetic energy spectrum of the generated flow field is consistent with the notion that migrating plankton can affect the large-scale structure of the water column despite their small individual size. Furthermore, real time flow visualizations reveal the formation of large-scale eddies, demonstrating the existence of an inverse cascade of kinetic energy from the small scale of individual swimmers to larger scales at which mixing can be efficient.

Section II describes the laser guidance system and measurement techniques, including the protocol for achieving DVM on demand. Results including the measured velocity fields, kinetic energy spectra, and qualitative flow visualizations are presented in Sec. III. Finally, the implications and limitations of the present findings in the context of local and global ocean dynamics are discussed in Sec. IV.

\section{EXPERIMENTAL METHODS}

\section{A. Test organism}

Artemia salina, a species of brine shrimp, was used as a model organism for these experiments. The dormant cyst of this crustacean hatches into a nauplius larva and grows to juvenile and adult stages reaching up to $15 \mathrm{~mm} .{ }^{22}$ Juveniles of this species have 11 pairs of legs and swim by beating their appendages in a metachronal wave. Two-week old juveniles (Mariculture Tech Intl.) with a body length of approximately $5 \mathrm{~mm}$ were used in all experiments (Figure 1).

An important feature of A. salina for the present experiments is their behavioral response to specific ranges of light wavelength and intensity. This reaction, known as phototaxis, is thought to trigger vertical motion in the ocean. By sensing light conditions within the water column, zooplankton that undergo DVM are able to adjust their position with respect to the ocean surface. In the present experiment, the phototactic capability of the plankton is exploited to achieve collective vertical motion of aggregations of swimming A. salina through a combination of luminous stimuli, as described below.

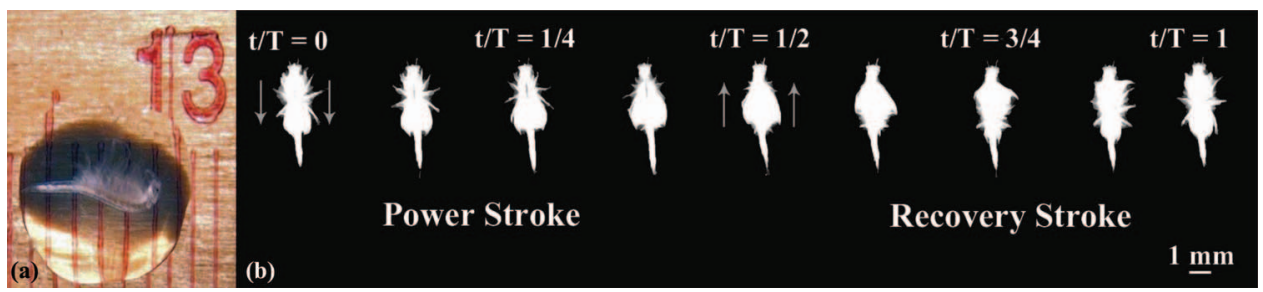

FIG. 1. Test organism. (a) Image of a two-week old juvenile A. salina in a water droplet. Ruler in background indicates millimeter units. (b) Time series showing the power and the recovery strokes that form the metachronal wave of a two-week old A. salina. The complete cycle ranges from $t / T=0$ to $t / T=1$, where time $t$ is measured from the beginning of the swimming cycle and $T$ is duration of the swimming cycle. The direction of appendage motion is indicated by the gray arrows at the beginning of each stroke. 

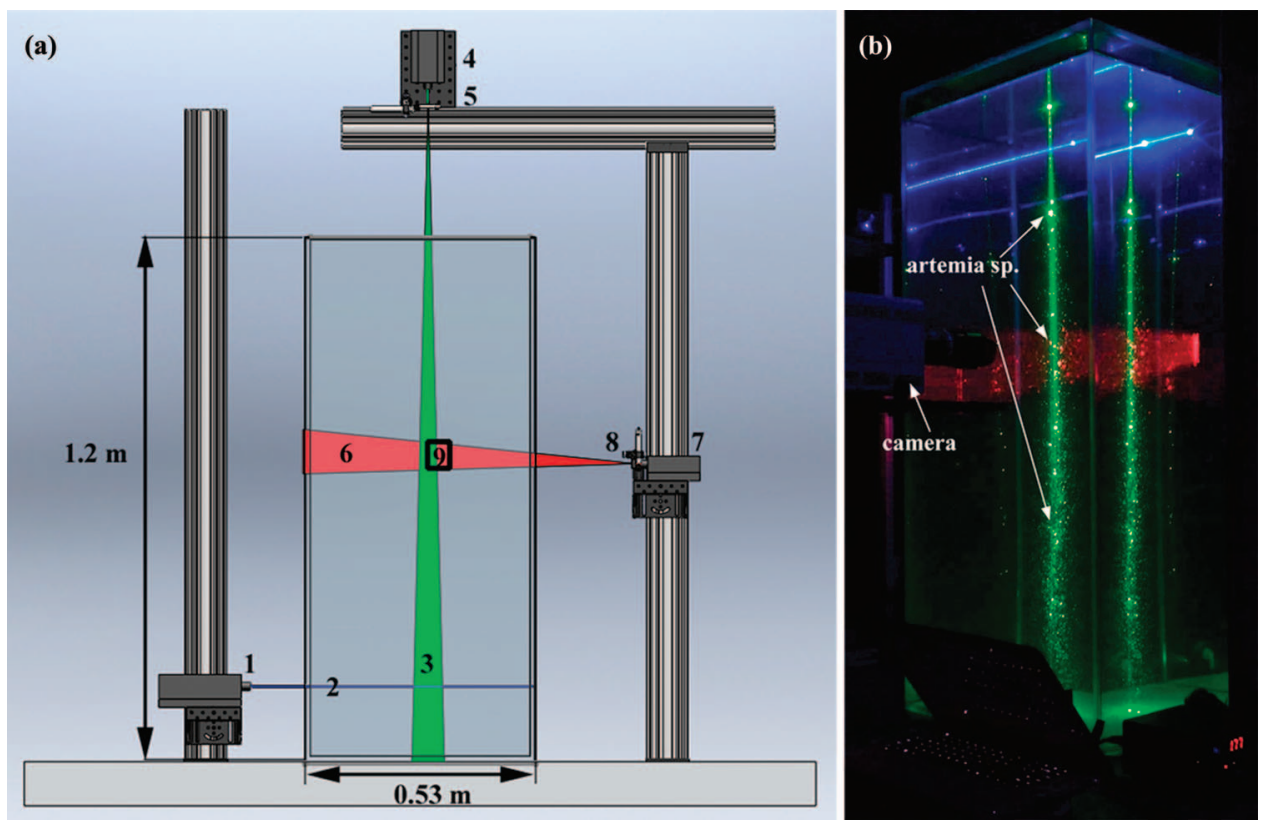

FIG. 2. Laser guidance system for induction and control of vertical migrations of plankton aggregations. (a) The setup consists of a $447 \mathrm{~nm}$ laser (1) that generates a horizontal blue laser beam (2), which is aligned with a vertical green laser sheet (3), generated by a $532 \mathrm{~nm}$ laser (4), and a $-25 \mathrm{~mm}$ focal length cylindrical lens (5), and introduced from above the tank. A vertical, red laser sheet (6) is introduced from the side of the tank and is produced using a $635 \mathrm{~nm}$ laser (7) and a $-3.9 \mathrm{~mm}$ focal length cylindrical lens (8). PIV measurements are recorded within the overlap region (9). (b) Photo of the setup during a migration event, after the $447 \mathrm{~nm}$ laser has completed its vertical traverse of the tank. The image was captured at an angle, producing duplicate reflections of the lasers in the two visible sides of the tank. See the corresponding video of the experiment. (Multimedia view) [URL: http://dx.doi.org/10.1063/1.4895655.1].

Following methods in the literature, the phototactic behavior of A. salina was evaluated by systematically testing different wavelengths of light to identify ranges of maximum positive response. ${ }^{23}$ Decapsulated eggs (Cabrillo Marine Aquarium, San Pedro, CA) were hatched in the laboratory and raised to $48 \mathrm{~h}$ old. Tests were performed using a 7520 beam $\mathrm{lm}$ spotlight (Source Four 405-1). A series of gel filters mounted in front of the spotlight was used to span the visible light spectrum. The nauplii were observed to be positively phototactic to the blue-green portion of the color spectrum and neutral in their response to red. Furthermore, by comparing the nauplii distribution with respect to the light source position, it was observed that the strongest positive attraction occurred for wavelengths ranging from $420 \mathrm{~nm}$ to $500 \mathrm{~nm}$. Past this maxima in directional behavior, the phototactic response was found to decrease along the green region up to the red portion of the visible light spectrum, where animals were found to be impartial to the stimulus. Further tests with juveniles and adults showed that the strength of the directional response decreased as animals aged, but remained constant within the duration of each life cycle stage (one-three weeks for juveniles). Nonetheless, the range of wavelengths for which attraction occurred remained constant throughout the life cycle.

\section{B. Experimental setup}

Based on the results of Sec. II A, an experimental apparatus was designed to induce collective vertical motion of A. salina. As shown in Figure 2, the experimental setup consists of a $1.2 \mathrm{~m} \mathrm{H} \times$ $0.53 \mathrm{~m} \mathrm{~W} \times 0.53 \mathrm{~m} \mathrm{~L}$ acrylic tank and a newly developed three-laser guidance system that artificially stimulates animal migration. The dimensions of the container were sufficiently large to avoid wall effects.

As described in Sec. II A, the phototactic behavior of A. salina dictated the design of the apparatus. Collective animal motion was triggered and controlled by means of luminescent signals from a $2 \mathrm{~W}, 447 \mathrm{~nm}$ (blue) laser and a $1 \mathrm{~W}, 532 \mathrm{~nm}$ (green) laser. The blue laser beam functioned 
as a cue for vertical migration to the aggregation, given that its intensity and wavelength matched the range of maximum positive phototaxis. This laser was mounted on a custom vertical traverse (Parker Hannifin) that controlled the position and vertical speed of the beam via a software interface (ACR-View 6).

The introduction of a second light stimulus was needed to position the migrating aggregation within the central region of the tank as well as constrain animal motion within a two-dimensional plane to enable PIV measurements. The reflection of the laser beam on the side wall of the tank where it is introduced produced a bright spot on the surface, which resulted in animal aggregation directly by the wall. Thus, even though the blue light stimulus resulted in vertical collective motion by itself, a second signal was needed to center the position of the organisms during vertical migration. The introduction of a vertical green laser sheet from above created a brighter region at the center of the column, resulting from the alignment of the green laser sheet with the blue laser beam. This combination of light signals ensured that the organisms swam vertically in the center of the tank. Moreover, the width of the green laser sheet (i.e., its horizontal span) determined the inter-organism spacing due to the behavioral preference of the organisms to swim within regions illuminated by stimuli triggering positive phototaxis. In this way, the organisms aggregated within the planar section inscribed by the green laser sheet and migrated following the steady vertical motion of the blue laser beam. Furthermore, minimal sheet divergence resulted in nearly constant animal spacing with height.

The resulting three-dimensional flow was measured within the two-dimensional plane shown in Figure 2 via PIV. For this purpose, a horizontal laser sheet was generated by a $1 \mathrm{~W}, 635 \mathrm{~nm}$ (red) laser, and aligned with the green laser sheet. The neutral behavior of the organisms towards the red portion of the light spectrum allowed for non-intrusive measurements of the velocity field during animal migration.

\section{Measurement protocol}

Experiments were conducted in $15 \mathrm{ppt}$ saltwater at room temperature, pre-mixed with $13 \mu \mathrm{m}$ silver-coated hollow glass spheres for PIV (Potters Industries Inc.). Prior to each test, the organisms were kept in aerated glass beakers with a low flow rate and water salinity set to match the conditions in the test tank. After this acclimatization process, test organisms (sample size range of $6500 \pm$ 4800) were drained from the glass beakers and introduced at the top of the tank using a fine mesh fish net.

With the laboratory lights turned off, the blue laser was positioned at the bottom of the water tank and subsequently activated. The organisms swam downward in phototactic response, gathering around the blue laser beam (Figure 3(a)). Reflection of the incoming blue laser beam at the side of the water tank resulted in greater animal concentration at the wall. Once the organisms were distributed around the blue laser beam, the green laser was activated, generating a narrow vertical green laser sheet. Simultaneously, the blue laser beam was momentarily deactivated, in order to induce horizontal animal motion towards the green laser sheet in the center of the tank (i.e., away from the brightest portion of the blue beam at the wall). As the organisms were evenly distributed at the center of the water column, the blue laser beam was reactivated and set to translate upward at $1 \mathrm{~cm} / \mathrm{s}$ (Figure 3(b)). A microcontroller (Acrobotic Industries) mounted on the vertical traverse and located at the initial position of the blue laser triggered a high-speed camera (Photron Ultima APX-RS), which was oriented perpendicular to the plane to be illuminated by the red laser sheet. The red laser was then turned on to generate a continuous red laser sheet. Video was recorded at midheight within the overlap region shown in Figure 3(c). The full measurement protocol is recorded in a video in Figure 2 (see the supplementary material) ${ }^{27}$ (Multimedia view).

Following the protocol delineated above, three sets of measurements were obtained by triggering the camera according to three time delays $\left(\tau_{1}=0 \mathrm{~s}, \tau_{2}=47 \mathrm{~s}\right.$, and $\left.\tau_{3}=67 \mathrm{~s}\right)$, corresponding to different stages of the migration. The first delay captured measurements of the flow conditions in the tank prior to vertical migration, as the organisms gathered within the blue beam at the bottom of the container (Figure 3(a)). Once this control data set was gathered, measurements were collected for either the first or second time delay, corresponding to the beginning or middle of the migration, respectively. The beginning and middle of the migration were defined to start when the first 

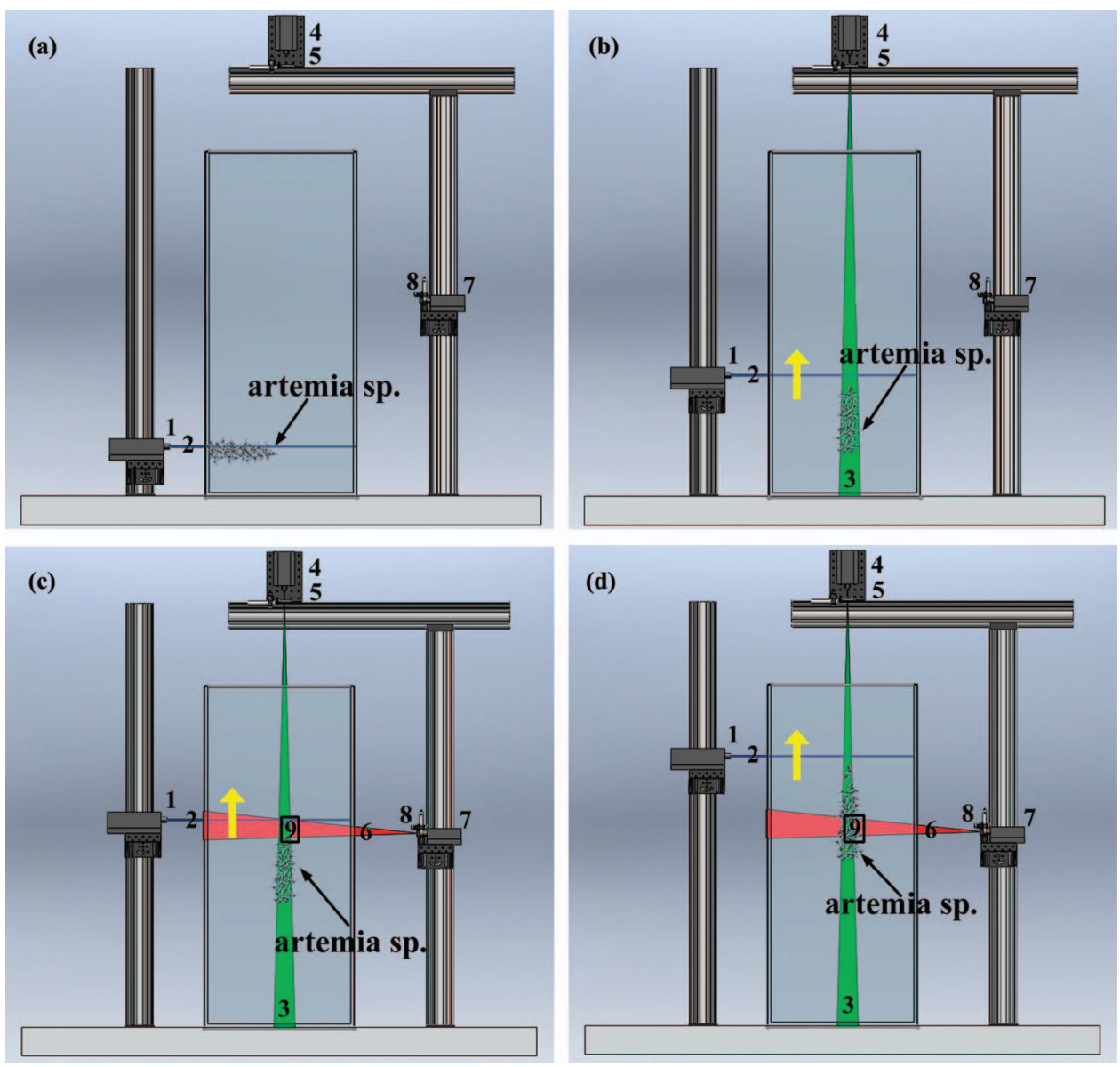

FIG. 3. Experimental procedure. Prior to triggering vertical motion, the organisms were attracted towards the bottom of the tank (a). Subsequently, vertical motion was triggered by activating the green laser sheet and translating the blue beam upwards (b). Measurements were taken for different stages in the migration: the stage referred to as the beginning of the migration occurred when the first organisms in the aggregation reached $1 / 2$ of the height of the tank (c), whereas the middle of the migration occurred when the first migrating organisms have reached $3 / 4$ of the height of the tank (d).

organisms in the aggregation reached approximately $1 / 2$ and $3 / 4$ of the height of the tank, respectively (Figures 3(c) and 3(d)). A total of 10 vertical migrations were measured: 5 recorded for the second delay and 5 for the third delay, each with a corresponding control measurement at the first time delay.

Qualitative flow visualizations were collected using both the square cross-section tank described above and an earlier tank design with circular cross-section within the same overlap region shown in Figure 2. The pathline measurements presented below were collected using the earlier tank design.

\section{PIV processing and spectral velocity tensor calculation}

PIV measurements were recorded at $250 \mathrm{fps}$ within the $2.8 \times 2.8 \mathrm{~cm}^{2}$ field of view depicted in Figure 2 at the described time delays. Raw PIV images were processed using a commercial code (DaVis 9, LaVision $\mathrm{GmbH}$ ). No filters were applied to mask the shape of the organisms, as the spatial resolution of the raw images was sufficient to resolve the flow very close to the animal body. The velocity fields were computed by cross-correlating image pairs separated by $\Delta t=0.06 \mathrm{~s}$. Image interrogation was conducted by iterating twice with a multi-pass method of decreasing window size from $64 \times 64$ pixels to $32 \times 32$ with a $50 \%$ overlap, yielding a spatial separation of $0.4 \mathrm{~mm}$ between 
velocity vectors. Post-processing was accomplished by applying a universal outlier detection test at the end of the routine.

After instantaneous velocity fields were computed at every time step, the fluctuating velocity fields were computed by Reynolds decomposition of each velocity component at each node of the velocity field

$$
u_{i}^{\prime}=u_{i}-\overline{u_{i}}, \quad i=(1,2),
$$

where an overbar denotes the temporal mean of the velocity component and a prime denotes the fluctuating velocity component. The components of the two-point velocity correlation tensor were computed at each node in the velocity grid according to the following expression for $|\mathbf{r}| \epsilon[0,3.9] \mathrm{cm}$,

$$
\mathbf{R}_{i j}(\mathbf{r}) \equiv \overline{u_{i}^{\prime}(\mathbf{x}) u_{j}^{\prime}(\mathbf{x}+\mathbf{r})}, \quad i, j=(1,2) .
$$

Thereafter, the spectral velocity tensor $\phi_{i j}$, from which the kinetic energy spectrum can be inferred, was computed from the two-point velocity correlation according to

$$
\boldsymbol{\phi}_{i j}(\boldsymbol{\kappa})=\frac{1}{(2 \pi)^{3}} \iiint_{-\infty}^{\infty} \mathbf{R}_{i j}(\mathbf{r}) e^{-i \kappa \mathbf{r}} d \mathbf{r}, \quad i, j=(1,2),
$$

which was discretized by using the 2D Fast Fourier Transform in MATLAB within the spatial limits imposed by the field of view and the Nyquist limit (i.e., twice the PIV vector spacing).

\section{RESULTS}

As shown in Figure 4, the generated near-field flow of a single vertically-migrating organism was found to vary between its ventral and dorsal sides. During the power stroke of the propulsive cycle, the metachronal motion of the organism appendages resulted in a quasi-steady downward jet, which persisted during the entire swimming cycle (see Fig. 4(a)).

In contrast, upward fluid motion was observed to be induced by the ventral side during the recovery stroke and by the dorsal side during upward animal motion (Figure 4(b)). The net direction of near-field fluid transport over the swimming cycle was found to be downward (i.e., opposite to the swimming direction), in contrast with previous observations of jellyfish. ${ }^{9}$ However, both results are consistent with recent theoretical models ${ }^{24}$ that predict a dependence of the net direction of induced drift on the swimming mode of the organism. . $^{15,25,26}$

Complex fluid interactions occurred as two or more organisms came in close proximity during collective vertical migration (Figure 4(c)). As shown in Figures 5(a)-5(d), the downward jet
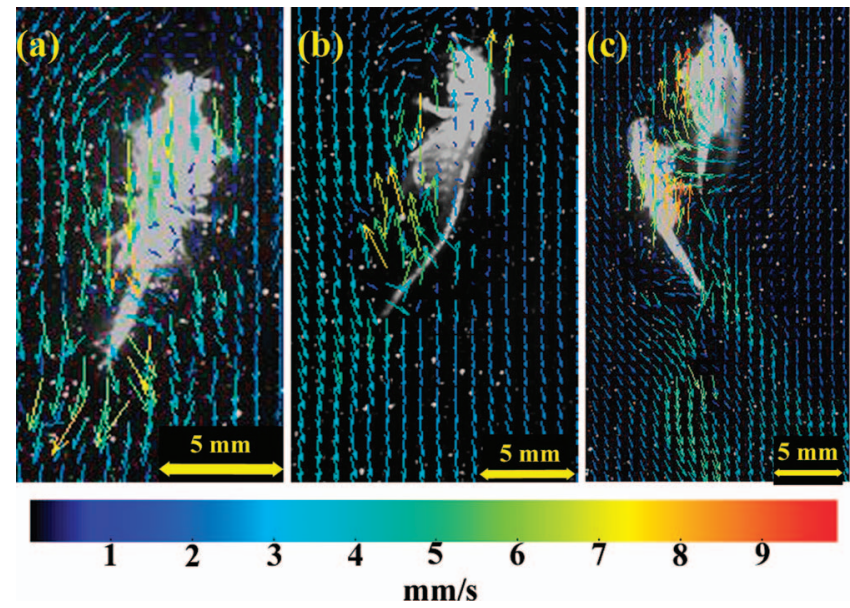

FIG. 4. Near-field flows during DVM. Near-field flows generated by the metachronal motion of the appendages of an individual organism (a), by the dorsal side of an individual organism (b), and by two organisms in close proximity (c). 

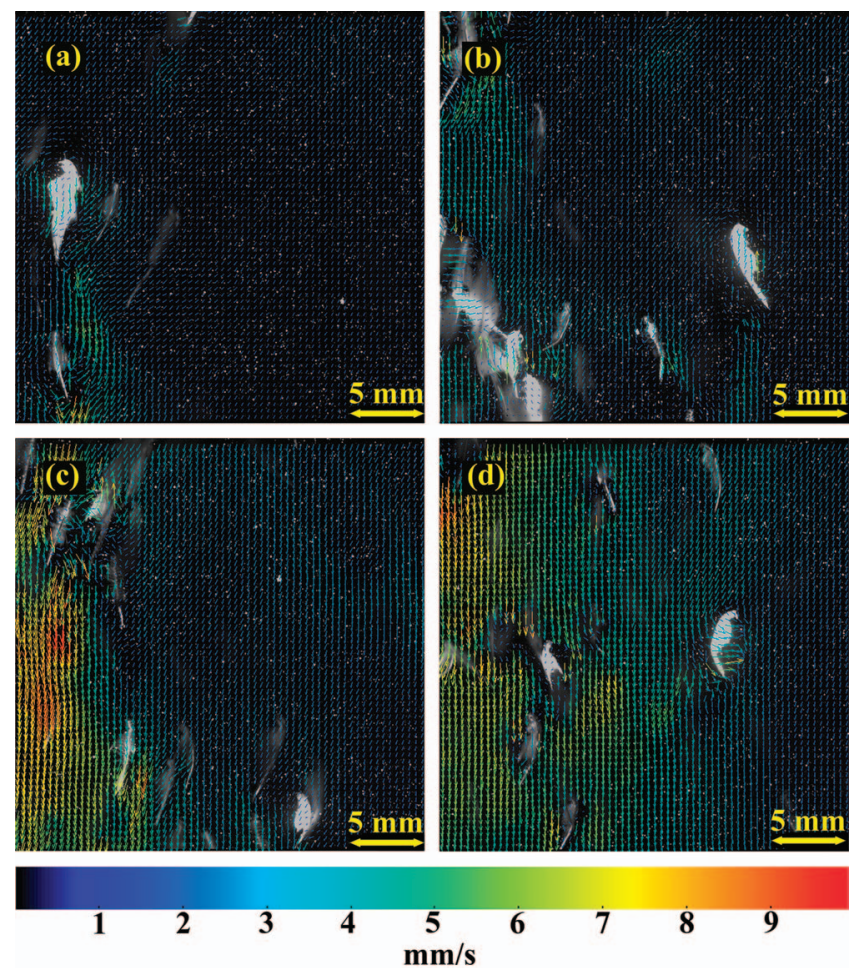

FIG. 5. Velocity field measurements during DVM. Time series for a representative set acquired with the second time delay $\left(\tau_{2}=47 \mathrm{~s}\right)$, which corresponds to the middle of the migration. The development of the downward jet is shown for the following times: $t=\tau_{2} \mathrm{~s}(\mathrm{a}), t=\tau_{2}+8 \mathrm{~s}(\mathrm{~b}), t=\tau_{2}+16 \mathrm{~s}(\mathrm{c}), t=\tau_{2}+24 \mathrm{~s}(\mathrm{~d})$.

generated by the active ventral side of a single organism dominated over the weak upward fluid drift induced along the passive dorsal side of the body and by the appendages during the recovery stroke. As a result, as the migration developed, collective animal motion incited a strong downward flow, characterized by a persistent, but spatially and temporally intermittent, downward jet that diverged over time (see Videos 1 and 2 of the supplementary materia ${ }^{27}$ ).

The spectral velocity tensor $\boldsymbol{\phi}_{i j}(\boldsymbol{\kappa})$ was computed from the measured PIV velocity fields for each of the three time delays from the beginning of the migration as described in Sec. II D. It was observed that as the migration progressed, kinetic energy increased within the entire measurable range of length scales (Figure 6(a)). The evolution of the spectrum with wavenumber for all cases showed that the energy produced by the collective vertical motion of small swimmers is introduced into the flow at scales considerably larger than the body length of a single organism. Moreover, the rate at which energy was transferred among different wavenumbers remained constant following a $\kappa^{-1}$ power law.

The measured shape of the kinetic energy spectrum is consistent with the power law observed in shear-dominated flows. ${ }^{28,29}$ Energy production occurs at length scales comparable to the organism appendages, as individual swimmers propel themselves upward by generating an unsteady shearing flow in the direction opposite to their motion. As illustrated in Figure 6(b), the flow speed was highest within the central region of the laser sheet and decreased towards the edge of the laser sheet, matching the concentration of organisms across the tank. Hence, as the migration develops, nearfield flow interactions among the organisms result in a spatially and temporally growing jet. Spatial gradients of velocity in the region of high shear at the boundary of the jet trigger a Kelvin-Helmholtz instability, which leads to the generation of eddies within the water column that are much larger than the individual organisms.

Qualitative flow visualizations of a larger field of view than that used for PIV at the same vertical position confirmed the formation of eddies next to the downward jet, which is consistent 

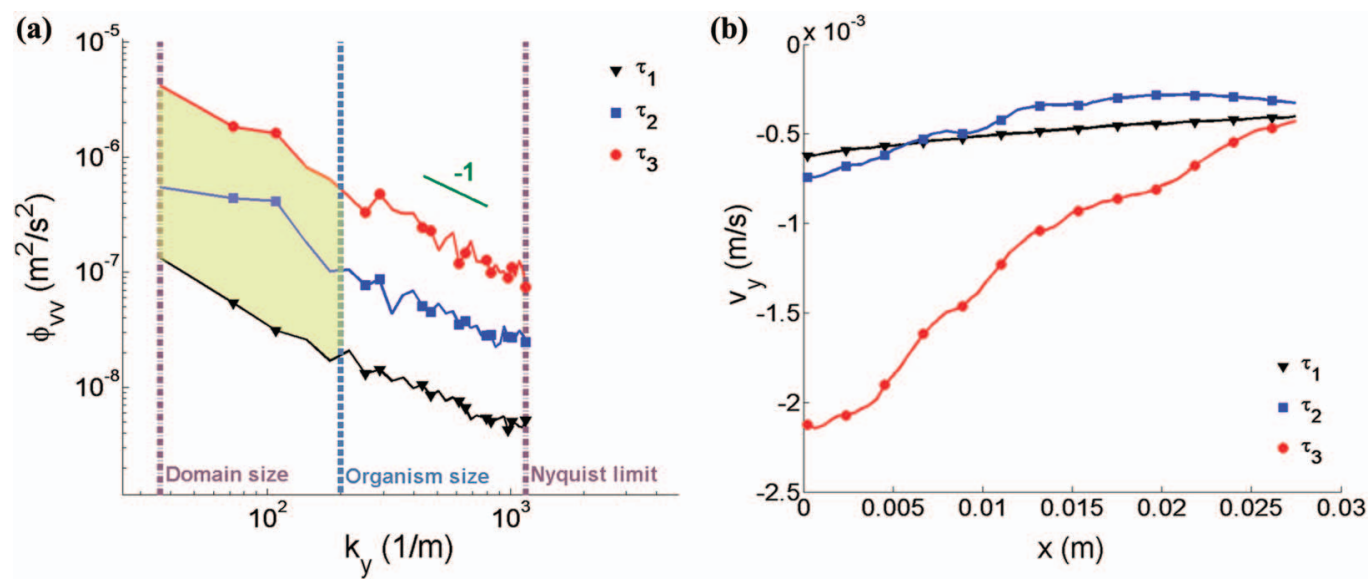

FIG. 6. Kinetic energy spectra and vertical velocity profiles during vertical migration. (a) Vertical component of the velocity spectrum tensor for the three time delays corresponding to different stages in the migration at which data were acquired. The vertical lines indicate the wavenumbers corresponding to the domain size (i.e., field of view), organism size, and the Nyquist limit (i.e., twice the PIV vector spacing). The yellow shaded region indicates increasing kinetic energy at length scales greater than the individual animal size (i.e., wavenumbers smaller than that corresponding to the individual animal size). (b) Mean vertical velocity along a horizontal transect across the center of the field of view for each stage of the migration. The left edge of the field of view approximately matches the central region of the green laser sheet (see Figure 2).

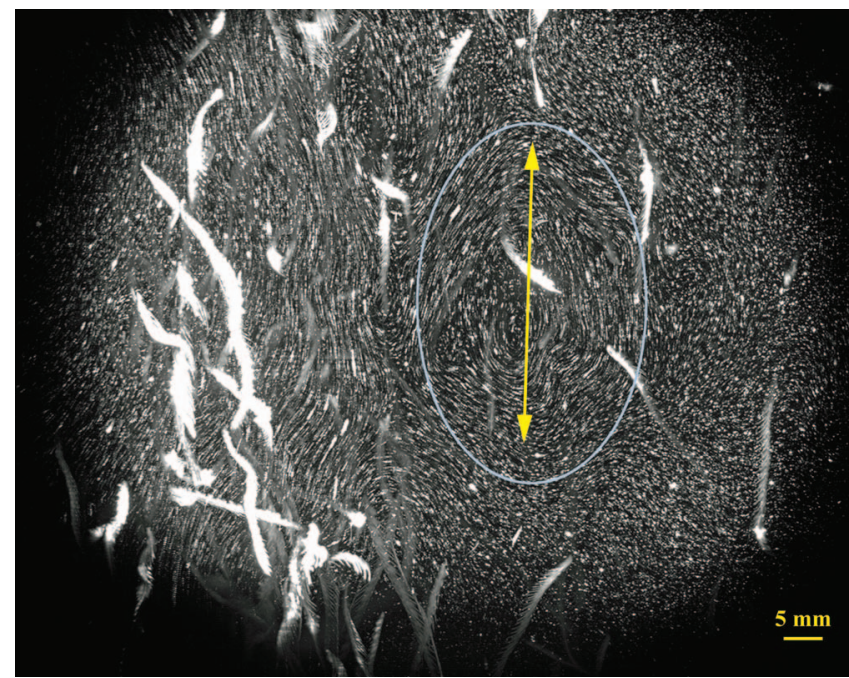

FIG. 7. Flow visualization showing the pathlines of PIV particles seeded in the water as well as the swimming organisms The depicted particle and animal streaks are the result of overlaying a 30-s sequence of raw PIV images recorded during the middle of a vertical migration. The generated large-scale flow structures next to the downward jet (e.g., blue oval) are significantly larger than the individual organisms. The vignetting of the image is due to the narrow-band laser filter on the camera, which becomes apparent when zoomed out to capture the larger-scale flow structures.

with the onset of a Kelvin-Helmholtz instability (Figure 7). This observation matches a linear stability analysis of the flow, which predicts that the length scale of the generated flow structures is at least an order of magnitude greater than the body size of a single organism (see Sec. IV).

\section{DISCUSSION AND CONCLUSIONS}

An experimental setup that enables controlled studies of fluid transport by vertically migrating plankton aggregations has been developed and implemented. Time-resolved PIV measurements indicate the formation of large-scale flow structures in the vicinity of aggregations of small, vertically 
migrating organisms. The corresponding kinetic energy spectrum exhibits an increase of energy over all measurable length scales including, most importantly, length scales larger than the size of an individual organism. We hypothesize that this large-scale transport is related to the non-uniform animal concentration in the water column, which was imposed by the finite width of the green laser sheet in the laboratory apparatus. This is consistent with the DVM process as it occurs in the ocean, wherein aggregations of zooplankton are also found to form non-uniform patches as seen in photographic data as well as in recent glider measurements. ${ }^{30,31}$

As illustrated in Figures 5 and 6, a downward jet became the dominant feature of the flow field as the migration progressed in time. The shear flow generated by an unsteady jet may be considered as the result of the interaction of unstable waves. It has been shown that linear stability analysis yields a good description of the initial development of shear layers such as those occurring in jets. ${ }^{32}$ Thus, knowledge of the shear layer shape, quantified by the momentum thickness, provides insight into the length scale of the eddies that form via the Kelvin-Helmholtz instability. The Strouhal frequency of the most unstable jet mode is given by $S t_{n}=f_{n} \theta_{0} U^{-1}=0.032$, where $f_{n}$ is the natural frequency of the unstable mode, $\theta_{0}$ is the momentum thickness of the shear layer, and $U$ is the mean velocity in the shear layer. ${ }^{32}$ The measured momentum thickness in the jet formed during vertical migration was $\theta_{0} \approx 5 \mathrm{~mm}$; hence, the predicted length scale of eddies formed due to the associated Kelvin-Helmholtz instability is $U f_{n}^{-1} \approx\left(10^{-1}\right) \mathrm{m}$, which is significantly larger than the individual animal size $(\approx 5 \mathrm{~mm})$.

These findings demonstrate the existence of an inverse cascade through which energy is introduced into the flow at the scale of individual swimmers and is subsequently transferred-via the collective formation of intermittent jets that are Kelvin-Helmholtz unstable-to larger scales at which mixing can occur efficiently.

Further studies of collective animal migrations in a stably stratified fluid are needed to investigate the evolution of the observed Kelvin-Helmholtz instability in the presence of restoring buoyancy effects, in order to properly assess the contribution of DVM to local and global oceanic processes. We hypothesize that the evolution of Kelvin-Helmholtz instabilities at the boundaries of the intermittent jets within a DVM could trigger Rayleigh-Taylor instabilities across surfaces of constant density and the formation of internal waves along those surfaces. Both processes could potentially result in large mixing eddies, which may explain previous findings of density overturns after krill migration in the ocean. ${ }^{33}$ Moreover, the additional presence of a Rayleigh-Taylor mode could imply an increase of the estimated limit of the mixing efficiency from the observed value of 0.2 for shear instabilities up to 0.5 , which has been measured for mixing by Rayleigh-Taylor unstable flows. ${ }^{34}$

\section{ACKNOWLEDGMENTS}

The authors gratefully acknowledge funding from the US-Israel Binational Science Foundation, the Office of Naval Research, and the National Science Foundation.

\footnotetext{
${ }^{1}$ M. E. Huntley and M. Zhou, "Influence of animals on turbulence in the sea," Mar. Ecol. Prog. Ser. 273, 65-79 (2004).

${ }^{2}$ W. K. Dewar, R. J. Bingham, R. L. Iverson, D. P. Nowacek, L. C. S. Laurent, and P. H. Wiebe, "Does the marine biosphere mix the ocean?" J. Mar. Res. 64, 541-561 (2006).

${ }^{3}$ E. Kunze, J. F. Dower, I. Beveridge, R. Dewey, and K. P. Bartlett, "Observations of biologically generated turbulence in a coastal inlet," Science 313, 1768-1770 (2006).

${ }^{4}$ K. Katija, "Biogenic inputs to ocean mixing," J. Exp. Biol. 215, 1040-1049 (2012).

${ }^{5}$ C. M. Moore, M. M. Mills, K. R. Arrigo, I. Berman-Frank, L. Bopp, P. W. Boyd, E. D. Galbraith, R. J. Geider, C. Guieu, S. L. Jaccard, T. D. Jickells, J. L. Roche, T. M. Lenton, N. M. Mahowald, E. Marañón, I. Marinov, J. K. Moore, T. Nakatsuka, A. Oschlies, M. A. Saito, T. F. Thingstad, A. Tsuda, and O. Ulloa, "Processes and patterns of oceanic nutrient limitation," Nature Geo. 6, 701-710 (2013).

${ }^{6}$ K. L. Denman and A. E. Gargett, "Biological-physical interactions in the upper ocean: The role of vertical and small scale transport processes,” Ann. Rev. Fluid Mech. 27, 225-255 (1995).

${ }^{7}$ W. Munk and C. Wunsch, "Abyssal recipes II: Energetics of tidal and wind mixing," Deep Sea Res. I 45, 1977-2010 (1998).

${ }^{8}$ A. W. Visser, "Biomixing of the oceans?" Science 316, 838-839 (2007)

${ }^{9}$ K. Katija and J. O. Dabiri, "A viscosity-enhanced mechanism for biogenic ocean mixing," Nature (London) 460, 624-626 (2009).
} 
${ }^{10}$ T. R. Osborn, "Estimates of the local rate of vertical diffusion from dissipation measurements," J. Phys. Oceanogr. 10, 83-89 (1980).

${ }^{11}$ S. A. Thorpe, The Turbulent Ocean (Cambridge University Press, 2005).

${ }^{12}$ N. S. Oakey, "Determination of the rate of dissipation of turbulent energy from simultaneous temperature and velocity shear microstructure measurements," J. Phys. Oceanogr. 12, 256-271 (1982).

${ }^{13}$ C. Darwin, "Note on hydrodynamics," Proc. Camb. Phil. Soc. 49, 342-354 (1953).

${ }^{14}$ This excludes the Stokes flow limit, i.e., large scale fluid transport may occur provided the organisms are sufficiently large to swim relative to the local flow.

${ }^{15}$ G. Subramanian, "Viscosity-enhanced bio-mixing of the oceans," Curr. Sci. 98, 1103-1108 (2010).

${ }^{16}$ C. Noss and A. Lorke, "Zooplankton induced currents and fluxes in stratified waters," Water Qual. Res. J. Can. 47, 276-286 (2012).

${ }^{17}$ N. A. Hill and T. J. Pedley, "Bioconvection,” Fluid Dyn. Res. 37, 1-20 (2005).

${ }^{18}$ D. L. Koch and G. Subramanian, "Collective hydrodynamics of swimming microorganisms: Living fluids," Ann. Rev. Fluid Mech. 43, 637-659 (2011).

${ }^{19}$ J. O. Dabiri, "Role of vertical migration in biogenic ocean mixing," Geophys. Res. Lett. 37, L11602, doi:10.1029/2010GL043556 (2010).

${ }^{20}$ S. Rousseau, E. Kunze, R. Dewey, K. Bartlett, and J. Dower, "On turbulence production by swimming marine organisms in the open ocean and coastal waters," J. Phys. Oceanogr. 40, 2107-2121 (2010).

${ }^{21}$ C. Noss and A. Lorke, "Direct observation of biomixing by vertically migrating zooplankton," Limnol. Oceanogr. 59, 724-732 (2014).

${ }^{22}$ Encyclopedia Britannica Online, s.v. "Brine shrimp," see http://www.britannica.com/EBchecked/topic/79674/brine-shrimp (accessed August 26, 2014).

${ }^{23}$ C. M. Owens, "Behavioral assay to determine photosensitivity of early-stage nauplii of the brine shrimp Artemia franciscana," B.S. thesis (Wagner College, 2002).

${ }^{24}$ D. O. Pushkin, H. Shum, and J. M. Yeomans, "Fluid transport by individual microswimmers," J. Fluid Mech. 726, 5-25 (2013).

${ }^{25}$ A. M. Leshansky and L. M. Pismen, "Do small swimmers mix the ocean?," Phys. Rev. E 82, 025301(R) (2010).

${ }^{26}$ J. Thiffeault and S. Childress, "Stirring by swimming bodies," Phys. Lett. A 374, 3487-3490 (2010).

${ }^{27}$ See supplementary material at http://dx.doi.org/10.1063/1.4895655 for videos 1 and 2 showing the measured velocity fields at the beginning and middle of the migration, respectively, of representative vertical migrations.

${ }^{28}$ A. E. Perry, S. Henbest, and M. S. Chong, "A theoretical and experimental study of wall turbulence," J. Fluid Mech. 165, 163-199 (1986).

${ }^{29}$ J. Jimenez, "The physics of wall turbulence," Phys. A 263, 252-262 (1999).

${ }^{30}$ W. M. Hamner and P. P. Hamner, "Behavior of Antarctic krill (Euphausia superba): School, foraging, and antipredatory behavior," Can. J. Fish. Aquat. Sci. 57, 192-202 (2000).

${ }^{31}$ D. Guihen, S. Fielding, E. J. Murphy, K. J. Heywood, and G. Griffiths, "An assessment of the use of ocean gliders to undertake acoustic measurements of zooplankton: The distribution and density of Antarctic krill (Euphausia superba) in the Weddell Sea," Limnol. Oceanogr.: Methods 12, 373-389 (2014).

${ }^{32}$ C. Ho and P. Huerre, "Perturbed free shear layers," Ann. Rev. Fluid Mech. 16, 365-424 (1984).

${ }^{33}$ E. Kunze, J. F. Dower, R. Dewey, E. A. D’Asaro, and A. W. Visser, “Mixing it up with krill (with response)," Science 318, 1239 (2007).

${ }^{34}$ A. G. W. Lawrie and S. B. Dalziel, "Rayleigh-Taylor mixing in an otherwise stable stratification," J. Fluid Mech. 688, 507-527 (2011). 